\title{
The Hankel determinant of exponential polynomials*
}

\author{
RICHARD EHRENBORG
}

The Hankel matrix of order $n+1$ of a sequence $a_{0}, a_{1}, \ldots$ is the $n+1$ by $n+1$ matrix whose $(i, j)$ entry is $a_{i+j}$, where the indices range between 0 and $n$. The Hankel determinant of order $n+1$ is the determinant of the corresponding Hankel matrix, that is,

$$
\operatorname{det}\left(a_{i+j}\right)_{0 \leq i, j \leq n}=\operatorname{det}\left(\begin{array}{cccc}
a_{0} & a_{1} & \cdots & a_{n} \\
a_{1} & a_{2} & \cdots & a_{n+1} \\
\vdots & \vdots & \ddots & \vdots \\
a_{n} & a_{n+1} & \cdots & a_{2 n}
\end{array}\right) .
$$

The purpose of this note is two-fold. First we present evaluations of Hankel determinants of sequences of combinatorial interest related to partitions and permutations. Many such computations have been carried out by Radoux in his sequence of papers [2]-[5]. His proof methods include using a functional identity due to Sylvester and factoring the Hankel matrix. Second, unlike Radoux, we instead give bijective proofs to reveal the underlying structure of these identities.

A partition $\pi=\left\{B_{1}, \ldots, B_{k}\right\}$ of a finite set $S$ is a collection of non-empty subsets $B_{1}, \ldots, B_{k}$, called blocks, such that the blocks are disjoint and their union is the set $S$. Let $|\pi|$ denote the number of blocks in the partition $\pi$. The exponential polynomials $e_{n}(x)$ are defined by

$$
e_{n}(x)=\sum_{\pi} x^{|\pi|},
$$

where $\pi$ ranges over all partitions of an $n$-element set. A few properties of the exponential polynomials are (i) $e_{n}(1)$ is equal to the $n$th Bell number, (ii) $e_{n}(x)=\sum_{k=0}^{n} S(n, k) x^{k}$ where $S(n, k)$ is the Stirling number of the second kind, (iii) $e_{n}(x)=e^{-x}(x \cdot d / d x)^{n} e^{x}$, (iv) $\sum_{n \geq 0} e_{n}(x) t^{n} / n !=\exp \left(x\left(e^{t}-1\right)\right.$ ). For more on the properties of exponential polynomials, see [7, Section 13], which is [6, pp. 7-82].

Theorem 1 (Radoux [2]) The Hankel determinant of order $n+1$ of the exponential polynomials $e_{n}(x)$ is given by

$$
\operatorname{det}\left(e_{i+j}(x)\right)_{0 \leq i, j \leq n}=x^{(n+1) n / 2} \cdot \prod_{i=0}^{n} i ! .
$$

*This paper appeared in American Mathematical Monthly 107 (2000), 557-560. 
Setting $x=1$ in the identity gives the Hankel determinant of the Bell numbers. This case was mentioned by Martin Gardner in [1, p. 35].

Let $R_{0}, \ldots, R_{n}, C_{0}, \ldots, C_{n}$ be disjoint sets such that the cardinality of $R_{i}$ and $C_{i}$ is $i$. We call $R_{i}$ the $i$ th row set and $C_{i}$ the $i$ th column set. Moreover, assume that the set $R_{i}$ possesses a linear order. Let $S$ be the disjoint union of all the row and column sets. A partition $\pi$ of the set $S$ and a permutation $\sigma$ of the set $\{0,1, \ldots, n\}$ are called compatible if for all blocks $B$ in $\pi$ there exists an index $i$ such that $B \subseteq R_{i} \cup C_{\sigma(i)}$. Let $T$ denote the set of all pairs $(\sigma, \pi)$, where $\sigma$ is a permutation and $\pi$ is a compatible partition. Finally, let $(-1)^{\sigma}$ denote the sign of the permutation $\sigma$.

Proposition 2 The Hankel determinant of the exponential polynomials $e_{n}(x)$ has the following combinatorial interpretation:

$$
\operatorname{det}\left(e_{i+j}(x)\right)_{0 \leq i, j \leq n}=\sum_{(\sigma, \pi) \in T}(-1)^{\sigma} \cdot x^{|\pi|} .
$$

Proof: Consider the exponential polynomial $e_{i+j}(x)$ at row $i$ and column $j$ as a sum over all partitions of the set $R_{i} \cup C_{j}$. Then the determinant can be expanded as

$$
\sum_{\sigma}(-1)^{\sigma} \cdot\left(\sum_{\pi_{0}} x^{\left|\pi_{0}\right|}\right) \cdots\left(\sum_{\pi_{n}} x^{\left|\pi_{n}\right|}\right),
$$

where $\pi_{i}$ is a partition on the set $R_{i} \cup C_{\sigma(i)}$. By letting $\pi=\pi_{0} \cup \cdots \cup \pi_{n}$, we obtain a partition on the set $S$ that is compatible with the permutation $\sigma$. Moreover, any pair $(\sigma, \pi) \in T$ may be obtained in this way. Finally, observe that $|\pi|=\left|\pi_{0}\right|+\cdots+\left|\pi_{n}\right|$.

Proof of Theorem 1: Let $T_{1}$ be the subset of $T$ consisting of the pairs $(\sigma, \pi)$, where $\sigma$ is the identity permutation and each block $B$ of $\pi$ contains two elements, one from the row set $R_{i}$ and one from the corresponding column set $C_{i}$. Observe that

$$
\sum_{(\sigma, \pi) \in T_{1}}(-1)^{\sigma} \cdot x^{|\pi|}=x^{(n+1) n / 2} \cdot \prod_{i=0}^{n} i !,
$$

since $\pi$ consists of $(n+1) n / 2$ blocks and there are $i$ ! ways to choose a matching between $R_{i}$ and $C_{i}$.

Let $T_{2}=T-T_{1}$. We show that there exists an involution on the set $T_{2}$ that changes the sign of the permutation and keeps the number of blocks of the partition the same. Such an involution implies that

$$
\sum_{(\sigma, \pi) \in T_{2}}(-1)^{\sigma} \cdot x^{|\pi|}=0,
$$

and hence proves the theorem. 
For $(\sigma, \pi)$ in $T$ define $a_{i}$ to be the number of blocks $B$ that intersect both $R_{i}$ and $C_{\sigma(i)}$. Observe that $a_{i} \leq\left|R_{i}\right|=i$ and $a_{i} \leq\left|C_{\sigma(i)}\right|=\sigma(i)$. We call the $a_{i}$ 's the crossing numbers.

Consider first the case when all the crossing numbers $a_{i}$ are distinct. Then we have $a_{i}=i$ for all indices $i$ and we conclude that $\sigma$ is the identity permutation. Moreover, $\pi$ provides a matching between the sets $R_{i}$ and $C_{i}$. Hence this implies that $(\sigma, \pi)$ belongs to $T_{1}$.

Thus for $(\sigma, \pi)$ in $T_{2}$ we know that there exists a pair of indices $(j, k)$ such that $a_{j}=a_{k}$. Let $(j, k)$ be the least such pair in the lexicographic order. Let $\sigma^{\prime}$ be the permutation $\sigma^{\prime}(j)=\sigma(k), \sigma^{\prime}(k)=\sigma(j)$ and $\sigma^{\prime}(i)=\sigma(i)$ for $i \neq j, k$. Clearly, $(-1)^{\sigma^{\prime}}=-(-1)^{\sigma}$.

Now we construct a partition $\pi^{\prime}$ compatible with $\sigma^{\prime}$. Let $a=a_{j}=a_{k}$. Let $B_{1}, \ldots, B_{a}$ be the blocks of $R_{j} \cup C_{\sigma(j)}$ that intersect both $R_{j}$ and $C_{\sigma(j)}$. We can order these blocks in a canonical fashion according to the smallest element in the block intersected with $R_{j}$. This is why we assumed a linear order on the row set $R_{i}$. Similarly, let $D_{1}, \ldots, D_{a}$ be the blocks of $R_{k} \cup C_{\sigma(k)}$ that intersect both the row and the column set. Order these blocks in the same canonical fashion. Define new blocks by

$$
B_{i}^{\prime}=\left(B_{i} \cap R_{j}\right) \cup\left(D_{i} \cap C_{\sigma(k)}\right), \quad D_{i}^{\prime}=\left(D_{i} \cap R_{k}\right) \cup\left(B_{i} \cap C_{\sigma(j)}\right) .
$$

Let $\pi^{\prime}$ be the partition obtained from the partition $\pi$ by replacing the blocks $B_{1}, \ldots, B_{a}, D_{1}, \ldots, D_{a}$ with $B_{1}^{\prime}, \ldots, B_{a}^{\prime}, D_{1}^{\prime}, \ldots, D_{a}^{\prime}$. Observe that the two partitions $\pi$ and $\pi^{\prime}$ have the same number of blocks and $\pi^{\prime}$ is compatible with $\sigma^{\prime}$.

We claim that the map $(\sigma, \pi) \longmapsto\left(\sigma^{\prime}, \pi^{\prime}\right)$ is an involution. Observe that both pairs $(\sigma, \pi)$ and $\left(\sigma^{\prime}, \pi^{\prime}\right)$ have the same sequence of crossing numbers. Hence the map, when applied again, chooses the same pair $(j, k)$ and switches blocks $B_{i}^{\prime}, D_{i}^{\prime}$ with $B_{i}, D_{i}$. This proves that the map is an involution.

We now consider three other classes of polynomials, which we denote by $e_{n}^{[\leq 2]}(x), e_{n}^{[\geq 2]}(x)$ and $e_{n}^{[=2]}(x)$. Their definition is similar to that of the exponential polynomials $e_{n}(x)$, but we now restrict the sum to partitions with block sizes less than or equal to two, greater than or equal to two, and equal to two, respectively. Some properties of these polynomials are:

(i) $\sum_{n \geq 0} e_{n}^{[\leq 2]}(x) t^{n} / n !=\exp \left(x\left(t+t^{2} / 2\right)\right)$,

(ii) $\sum_{n \geq 0} e_{n}^{[\geq 2]}(x) t^{n} / n !=\exp \left(x\left(e^{t}-1-t\right)\right)$,

(iii) $\sum_{n \geq 0} e_{n}^{[=2]}(x) t^{n} / n !=\exp \left(x t^{2} / 2\right)$,

(iv) $e_{n}^{[\leq 2]}(1)$ is the number of involutions on an $n$-element set,

(v) $e_{n}^{[\geq 2]}(1)$ is the number of partitions on an $n$-element set without singleton blocks, and 
(vi) $e_{n}^{[=2]}(x)=(n-1) \cdot(n-3) \cdots 1 \cdot x^{n / 2}$ if $n$ is even, otherwise equal to zero.

By examing the proof of Theorem 1, we see that it also applies to partitions with block sizes less than or equal to two, greater than or equal to two, and equal to two. Hence we have the surprising result:

Theorem 3 The Hankel determinants of the polynomials $e_{n}^{[\leq 2]}(x), e_{n}^{[\geq 2]}(x)$, and $e_{n}^{[=2]}(x)$ are given by

$$
\operatorname{det}\left(e_{i+j}^{[\leq 2]}(x)\right)_{0 \leq i, j \leq n}=\operatorname{det}\left(e_{i+j}^{[\geq 2]}(x)\right)_{0 \leq i, j \leq n}=\operatorname{det}\left(e_{i+j}^{[=2]}(x)\right)_{0 \leq i, j \leq n}=x^{(n+1) n / 2} \cdot \prod_{i=0}^{n} i !
$$

The first Hankel determinant in Theorem 3 was evaluated by Radoux in [4].

By considering permutations $\pi$ on the set $S$ that are 'compatible' with permutations $\sigma$, one can give a bijective proof of the following theorem. We leave the details to the reader. The first Hankel determinant is classical, the second is due to Radoux [3].

Theorem 4 Let $D(n)$ denote the number of derangements of an n-element set, that is, the number of permutations without fixed points. The Hankel determinants of order $n+1$ of the matrices $[(i+j) !]$ and $[D(i+j)]$ are given by

$$
\operatorname{det}((i+j) !)_{0 \leq i, j \leq n}=\operatorname{det}(D(i+j))_{0 \leq i, j \leq n}=\left(\prod_{i=0}^{n} i !\right)^{2} .
$$

Acknowledgments. The author thanks Margaret Readdy for reading an earlier draft of this note. This work was done when the author was a member at the Institute for Advanced Study where he was supported by National Science Foundation, DMS 97-29992, and NEC Research Institute, Inc.

\section{References}

[1] M. Gardner, Fractal Music, Hypercards and more: Mathematical Recreations from Scientific American, W. H. Freeman and Company, New York, 1992.

[2] C. Radoux, Calcul effectif de certains déterminants de Hankel, Bull. Soc. Math. Belg. Sér. B 31 (1979) $49-55$.

[3] C. Radoux, Déterminant de Hankel construit sur des polynomes liés aux nombres de dérangements, European J. Combin. 12 (1991) 327-329. 
[4] C. Radoux, Déterminants de Hankel et théorème de Sylvester, in Séminaire Lotharingien de Combinatoire (Saint-Nabor, 1992), Publ. Inst. Rech. Math. Av., 498, Univ. Louis Pasteur, Strasbourg, 1992, pp. 115-122.

[5] C. Radoux, Addition formulas for polynomials built on classical combinatorial sequences, to appear in $J$. Comput. Appl. Math.

[6] G.-C. Rota, Finite Operator Calculus, Academic Press, Inc., New York, 1975.

[7] G.-C. Rota, D. Kahaner, and A. Odlyzko, On the foundations of combinatorial theory. VIII. Finite operator calculus, J. Math. Anal. Appl. 42 (1973) 684-760.

Royal Institute of Technology, S-100 44 Stockholm, Sweden, jrge@math.kth.se 